\title{
DIFFUSE, TRANSLUCENT \& HIGH-LATITUDE CLOUDS: THEORETICAL CONSIDERATIONS
}

\author{
E.F. van Dishoeck \\ Leiden Observatory \\ P.O. Box 9513 \\ 2300 RA Leiden, The Netherlands
}

\begin{abstract}
The relative importance of gas-phase, shock and grain-surface chemistry is discussed with reference to recent observational data. The relation between diffuse, translucent and high-latitude clouds is illustrated with models in which carbon is transformed from atomic $\left(\mathrm{C}^{+}, \mathrm{C}\right)$ to molecular $(\mathrm{CO})$ form with increasing extinction.
\end{abstract}

\section{Introduction}

The study of diffuse molecular clouds has traditionally attracted a lot of theoretical attention, since these clouds are thought to be the simplest clouds to model and thus to form the most severe test of the basic chemical networks. Compared with the astrochemistry meeting in Goa (see Black 1987), it appears at first sight that little progress has been made in the last six years. For example, apart from the recent discovery of interstellar NH (Meyer \& Roth 1991), there have been no new detections of molecules at optical wavelengths since that of $\mathrm{C}_{2}$ in 1977. The diffuse interstellar bands are still unidentified; there still is no satisfactory solution to the $\mathrm{CH}^{+}$problem; and the rate coefficient for the $\mathrm{C}^{+}+\mathrm{H}_{2}$ radiative association reaction - the reaction that is thought to initiate the carbon chemistry - is still uncertain by at least a factor of five.

Our understanding of diffuse clouds has increased, however, in other areas since 1985. First, we have expanded our view to different classes of diffuse clouds. In addition to the "classical" diffuse clouds with $A_{V} \lesssim 1 \mathrm{mag}$ observed with the Copernicus satellite, a significant body of optical data is now available for translucent $\left(A_{V}=1-5 \mathrm{mag}\right)$ and high-latitude clouds (van Dishoeck \& Black 1989). There has also been increased spectral resolution at optical wavelengths (see Crane, this volume) and extension of the observations to millimeter wavelengths (see Blitz, this volume). Finally, in terms of the rate coefficients, there has been enormous progress in our understanding of the photodissociation of the $\mathrm{CO}$ molecule. With this perspective in mind, a number of aspects of diffuse cloud chemistry will be discussed. The reader is referred to several recent reviews (van Dishoeck \& Black 1988a; Dalgarno 1988; van Dishoeck 1990) for more details.

\section{Physical structure}

In order to test the chemistry, an estimate of the physical parameters in the clouds is needed, since the reaction rates depend sensitively on them. The strength of the incident ultraviolet radiation field is particularly important since photodissociation and photoionization play a major role in the chemistry. These parameters are usually derived from the observed excitation of the various species, as summarized in Table 1 . The primary diagnostic in diffuse clouds is $\mathrm{H}_{2}$, for which lines arising from levels up to $J=7$ have been observed with 
TABLE 1. Diagnostics of Physical Conditions

\begin{tabular}{|c|c|c|c|c|c|c|c|}
\hline Species & & $\begin{array}{l}\text { Phys. Parameter } \\
\text { Probed }^{a}\end{array}$ & $\begin{array}{l}\text { Diffuse } \\
\text { Cloud }\end{array}$ & $\begin{array}{l}\text { Transl. } \\
\text { Cloud }\end{array}$ & $\begin{array}{l}\text { High-lat. } \\
\text { Cloud }\end{array}$ & Method & Ref. \\
\hline $\begin{array}{l}\mathrm{H}_{2} \text { low } \mathrm{J} \\
\mathrm{H}_{2} \text { high } \mathrm{J} \\
\mathrm{C}_{2} \text { low } \mathrm{J} \\
\mathrm{C}_{2} \text { high } \mathrm{J} \\
\mathrm{C}, \mathrm{C}^{+}, \mathrm{O} \\
\mathrm{CO} \text { low } \mathrm{J} \\
\\
\mathrm{CN} \text { low } \mathrm{J}\end{array}$ & $\mathbf{J}$ & $\begin{array}{c}\mathrm{T} \\
\mathrm{I}_{U V}, \text { formation } \\
\mathrm{T} \\
\mathrm{I}_{R} / \mathrm{n}_{H} \\
\mathbf{n}_{H}, \mathrm{~T} \\
\mathbf{n}_{H}, \mathrm{~T} \\
\mathrm{n}(\mathbf{e}), \mathbf{n}_{H}\end{array}$ & $\begin{array}{l}+ \\
+ \\
+ \\
+ \\
+ \\
+ \\
+ \\
+ \\
+\end{array}$ & $\begin{array}{l}- \\
- \\
+ \\
+ \\
- \\
- \\
+ \\
+ \\
+\end{array}$ & $\begin{array}{l}- \\
- \\
+ \\
+ \\
- \\
- \\
+ \\
+ \\
+\end{array}$ & $\begin{array}{l}\text { UV abs } \\
\text { UV abs } \\
\text { VIS abs } \\
\text { VIS abs } \\
\text { UV abs } \\
\text { UV abs } \\
\text { mm em } \\
\text { VIS abs } \\
\text { mm em }\end{array}$ & $\begin{array}{r}1,2 \\
1,2 \\
3 \\
3 \\
2,4 \\
2,5,6 \\
7,8 \\
9 \\
9\end{array}$ \\
\hline
\end{tabular}

${ }^{a} \mathrm{I}_{U V}=$ scaling factor for radiation at ultraviolet wavelengths $\lambda=912-1100 \AA ; \mathrm{I}_{R}=$ scaling factor in far-red $\lambda \approx 1 \mu \mathrm{m}$.

References: 1. Jura 1975; 2. van Dishoeck \& Black 1986; 3. van Dishoeck \& Black 1982; 4. Jenkins \& Shaya 1979; 5. Smith et al. 1978; 6. Crutcher \& Watson 1981; 7. van Dishoeck et al. 1991; 8. Falgarone et al. 1991; 9. Black \& van Dishoeck 1991a.

the Copernicus satellite. The lower $J$ levels are populated by collisions, and are therefore sensitive to temperature; however, the higher $J$ levels lie much too high in energy to be collisionally excited in gas with $T<100 \mathrm{~K}$. It is usually assumed that ultraviolet pumping is responsible for this excitation, so that the high- $J$ level population provides a direct measure of the strength of the ultraviolet radiation field between 912 and $1100 \AA$. However, it can also result from the $\mathrm{H}_{2}$ formation process on grains (Black \& Dalgarno 1977; van Dishoeck \& Black 1986; Wagenblast 1991), or from collisional excitation in a (shock-heated) layer of gas at $2000 \mathrm{~K}$. The $\mathrm{H}_{2}$ example thus demonstrates a common problem with diagnostics, namely that it is very rare that a single excitation mechanism dominates. Often a mixture of processes plays a role, and it is difficult to disentangle them.

Most of these diagnostics have been applied to the classical diffuse clouds studied with the Copernicus satellite. Unfortunately, for the more recently discovered translucent and highlatitude clouds, many of them are not available, in particular at ultraviolet wavelengths. Although the first observations of $\mathrm{C}, \mathrm{C}^{+}, \mathrm{O}$ and $\mathrm{CO}$ with the Hubble Space Telescope (HST) have just been published for the diffuse cloud toward $\xi$ Per with $A_{V} \approx 1$ mag (Savage et al. 1991; Cardelli et al. 1991; Smith et al. 1991), data for more reddened lines of sight have not yet been taken. Moreover, $H S T$ will not provide any information on the $\mathrm{H}_{2}$ rotational excitation, so that it is not possible to perform exactly the same analysis for these clouds as for the classical diffuse clouds. In particular, there are currently no constraints on the strength of the incident radiation field.

For the diffuse clouds such as the $\zeta$ Oph cloud, the various diagnostics give consistent results for the temperature and density structure within factors of two. The best fit to the data is for models in which the temperature decreases from $100 \mathrm{~K}$ or more at the edge to about $30 \mathrm{~K}$ in the center, and the density from about $100 \mathrm{~cm}^{-3}$ at the edge to several hundred $\mathrm{cm}^{-3}$ in the center. The ultraviolet radiation field is usually somewhat enhanced over the average field by a factor $I_{U V} \approx 2-4$, which is not unreasonable since most of the clouds lie in regions of active star formation such as Ophiuchus, in which there are plenty of young bright stars. This estimate of $I_{U V}$ refers to the field at the edge of the cloud. Deeper inside, the radiation is reduced because of absorption and scattering by grains and because of processes such as self-shielding (van Dishoeck 1988; Roberge et al. 1991). The shape of the radiation field at the shortest wavelengths also plays an 
important role. For the translucent and high-latitude clouds, the $\mathrm{C}_{2}$ and $\mathrm{CN}$ excitations give somewhat higher central densities, $n_{H} \approx 500-5000 \mathrm{~cm}^{-3}$, and lower temperatures, $T \approx 15-30 \mathrm{~K}$. The $\mathrm{CO}$ rotational excitation can be interpreted either with a low density, higher temperature solution (van Dishoeck et al. 1991), or with a very cold $(T=10 \mathrm{~K})$ and very dense $\left(n_{H}>10^{4} \mathrm{~cm}^{-3}\right)$ model (Falgarone et al. 1991). The former solution is consistent within the observational errors with the parameters derived from $\mathrm{C}_{2}$ and $\mathrm{CN}$.

\section{Steady-state models}

Given the set of physical conditions $n(z), T(z)$ and $I(z)$, the basic network of gas-phase ion-molecule reactions can be solved at each depth $z$ into the cloud. The availability of accurate reaction rate coefficients is a prerequisite for testing the chemistry. Other ingredients to the models are the elemental gas-phase abundances, which are constrained to fit the observations of the atomic lines where available, and the cosmic ray ionization rate $\zeta_{o}$, which is chosen such as to reproduce the observed $\mathrm{OH}$ abundance. The inferred value of $\zeta_{o}$ depends sensitively on the $\mathrm{H}_{3}^{+}$dissociative recombination rate coefficient at low temperatures (Amano 1990).

Consider as an example one of the simplest molecules, $\mathrm{CH}$. It is thought to be formed by the sequence starting with the radiative association of $\mathrm{C}^{+}$with $\mathrm{H}_{2}$ and is destroyed primarily by photodissociation. Thus, only few reactions are involved and roughly $n(\mathrm{CH}) \propto$ $k_{r a} n\left(\mathrm{C}^{+}\right) n\left(\mathrm{H}_{2}\right) / k_{p d}$ (Federman 1982). Models of diffuse clouds with the physical structure described in $\S 2$ can reproduce the observed $\mathrm{CH}$ column densities very well, so that $\mathrm{CH}$ has always been considered to be one of the strongest cases of support for the steady-state gas-phase chemistry. Yet there are several major uncertainties in this analysis. First, $k_{r a}$ is not known to better than a factor of five, in spite of several decades of theoretical and experimental work. The (indirect) experiments by Gerlich (1989) suggest $k_{r a} \approx 7 \times$ $10^{-16} \mathrm{~cm}^{3} \mathrm{~s}^{-1}$, whereas the theory by Smith (1989) gives $k_{r a} \approx(1-2) \times 10^{-15} \mathrm{~cm}^{3} \mathrm{~s}^{-1}$ at interstellar temperatures. Second, $n\left(\mathrm{C}^{+}\right)$depends on the adopted gas-phase carbon abundance, which is not known to better than $50 \%$. Also, the decrease of $n\left(\mathrm{C}^{+}\right)$with depth into the cloud depends on the physical structure ( $\S 4)$. Future observations of the semi-forbidden C II] line at $2325 \AA$ with $H S T$ may provide better constraints on the carbon abundance (Cardelli et al. 1991). Third, the model $\mathrm{CH}$ abundance is proportional to the density $n\left(\mathrm{H}_{2}\right)$; at higher densities, significantly more $\mathrm{CH}$ is produced than is observed. Fourth, the $\mathrm{CH}$ abundance is inversely proportional to the photodissociation rate, and thus $I_{U V}$, for which few constraints are available.

Given these uncertainties, it is not surprising that it is possible to fit the observational $\mathrm{CH}$ data with some combination of parameters. However, it would be impossible to establish whether all observed $\mathrm{CH}$ were produced by this mechanism; it is certainly possible that some fraction (up to $30 \%$ ) could by formed by another process, as seems implied by the high-resolution data of Lambert et al. (1990) (see also Crane, this volume). The same conclusion holds for each of the other molecules individually. The real test of the models is whether a consistent model can be built that reproduces the whole array of observational data for a cloud with the same set of parameters. Such comprehensive models have been developed by e.g. Black \& Dalgarno (1977), van Dishoeck \& Black (1986), Viala et al. (1988a) and Wagenblast \& Hartquist (1989), and are consistent with the observed abundances (and upper limits) of most species within factors of two. These same models can also reproduce the measured abundances in translucent and high-latitude clouds with only slightly different parameters, such as a lower incident radiation field and/or lower gas-phase carbon abundance (van Dishoeck \& Black 1989). However, there are a number of species for which the steady-state models fail. The best-known case is that of $\mathrm{CH}^{+}$, which the steady-state models underproduce by 1-2 orders of magnitude. Second, with the new CO 
photodissociation rate, the computed $\mathrm{CO}$ column densities are too low by factors of a few in some (but not all!) diffuse clouds. Third, the models fail to account for the observed $\mathrm{NH}$ abundance by an order of magnitude. Finally, they cannot reproduce the observed abundances of more complex species such as $\mathrm{H}_{2} \mathrm{CO}$ and $\mathrm{C}_{3} \mathrm{H}_{2}$ in the thicker translucent and high-latitude clouds. The CO problem is discussed in more detail in van Dishoeck \& Black (1988b) and van Dishoeck (1990). The other problems will be addressed in §5-7.

\section{Relation between diffuse, translucent \& high-latitude clouds}

Because the diffuse, translucent and high-latitude clouds are usually studied by different observational techniques (optical absorption, millimeter emission, IRAS $100 \mu \mathrm{m}$ ), it is often thought that they are of a different nature. The diagnostics discussed in $\S 2$ already suggest that the physical parameters in the translucent and high-latitude clouds do not differ significantly from those in the classical diffuse clouds: they may be somewhat denser, colder and/or exposed to less radiation, but probably not by large factors. In this section, it is illustrated that the observational division results mostly from the chemistry of CO in the transition zone between diffuse and dense interstellar clouds.

The crucial parameter in the $\mathrm{CO}$ chemistry is its destruction by photodissociation. Owing to the detailed laboratory work by Letzelter et al. (1987), Eidelsberg \& Rostas (1990) and Stark et al. (1991), the photoprocesses of CO are now fairly well characterized. The CO photodissociation occurs by line absorptions in the $912-1118 \AA$ range, where overlap with lines of $\mathrm{H}_{2}, \mathrm{H}$ and $\mathrm{CO}$ isotopes can occur. Detailed radiative transfer methods are therefore needed to compute the $\mathrm{CO}$ photodissociation rate as a function of depth into the cloud (van Dishoeck \& Black 1988b; Viala et al. 1988b). At the edge, all carbon is in atomic form as $\mathrm{C}^{+}$, but with increasing depth carbon is gradually transformed into $\mathrm{C}$ and $\mathrm{CO}$. Thus, the total column density of $\mathrm{CO}$ and its abundance with respect to $\mathrm{H}_{2}$ depend sensitively on the total extent of the cloud. In Figure 1, the $\mathrm{CO} / \mathrm{H}_{2}$ column density ratio is presented as a function of the total $\mathrm{H}_{2}$ column density or visual extinction. For diffuse clouds with $A_{V} \leqslant 1 \mathrm{mag}$, most of the carbon is still in the form of $\mathrm{C}^{+}$and $\mathrm{CO} / \mathrm{H}_{2} \approx 10^{-6}$. For thick, dense clouds with $A_{V} \approx 10 \mathrm{mag}$, all gas-phase carbon is transformed into $\mathrm{CO}$ and $\mathrm{CO} / \mathrm{H}_{2} \rightarrow 2[\mathrm{C}]_{\mathrm{gas}} /[\mathrm{H}]$, which is about $3 \times 10^{-4}$ in these models in which $40 \%$ of the solar carbon abundance is assumed to be in the gas phase. It is just in the region of the translucent clouds of $A_{V} \approx 1-5 \mathrm{mag}$ that the $\mathrm{CO} / \mathrm{H}_{2}$ column density ratio increases very rapidly by two orders of magnitude. Where exactly the transition occurs depends on the physical parameters of the cloud. For higher density and/or lower radiation field, it is shifted to lower $A_{V}$.

Figure 1 forms a convenient framework in which to characterize the various clouds. The observed $\mathrm{CO} / \mathrm{H}_{2}$ column density ratios of a number of diffuse and translucent clouds are included, and are found to follow the general trend. Since the detection limit for CO emission at millimeter wavelengths is $N(\mathrm{CO}) \approx \mathrm{a}$ few $\times 10^{14} \mathrm{~cm}^{-2}$ (or $\mathrm{CO} / \mathrm{H}_{2} \approx 10^{-6}$ ), it can be understood that Lada \& Blitz (1985) divided these same clouds into "CO poor" and "CO rich". Similarly, the high-latitude clouds can be fitted into this scheme. These clouds do not form a homogeneous sample, but actually cover the full range of $A_{V}$ from the very diffuse to dense interstellar clouds. Even within a single cloud, there may be significant structure. For example, there are the high-latitude clouds recognizable through their IRAS $100 \mu \mathrm{m}$ emission in which no $\mathrm{CO}$ millimeter emission has been detected (Blitz et al. 1990), which are indicated with "BBD90" in Figure 1. These clouds have $A_{V}<1$ mag and are probably similar to the diffuse clouds studied with Copernicus. On the other hand, high-latitude clouds such as MBM 12 show CO antenna temperatures similar to those found in thick, dense clouds such as TMC-1 (Pound et al. 1990). The majority of the high-latitude clouds detected by Magnani et al. (1985) probably fall in the middle region of the translucent clouds, where carbon is being transformed from atomic to molecular form. 


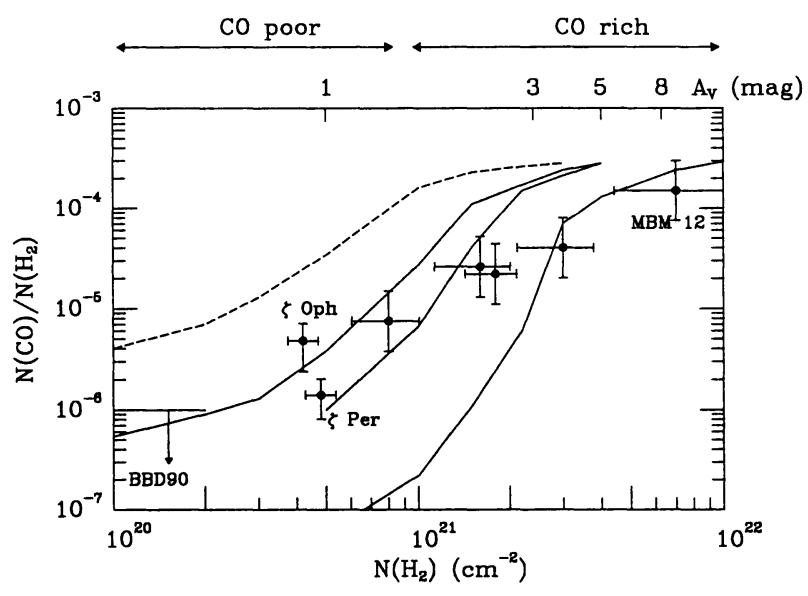

Figure 1. Computed and observed ${ }^{12} \mathrm{CO} / \mathrm{H}_{2}$ column density ratios as functions of total $\mathrm{H}_{2}$ column density (adapted from van Dishoeck \& Black 1988b). The full lines from left to right are model results for $I_{U V}=0.5,1.0$ and 10 , respectively, and $n_{H} \approx 500-2000$ $\mathrm{cm}^{-3}$. The dashed line is for models with $I_{U V}=1, n_{H}=10^{4} \mathrm{~cm}^{-3}$ and $T=10 \mathrm{~K}$. In such models, ${ }^{13} \mathrm{CO}$ is heavily fractionated, with $N\left({ }^{12} \mathrm{CO}\right) / N\left({ }^{13} \mathrm{CO}\right)=3-6$. Observed values for a number of diffuse, translucent and high-latitude clouds are included. The unlabelled points are for HD 210121, HD 169454, HD 154368 and HD 29647, respectively, from left to right. The limit marked BBD90 refers to Blitz et al. (1990).

In this regime, the column densities are very sensitive to slight changes in the physical parameters, so that the interpretation of the small-scale structure seen in the maps is not obvious, as discussed for the high-latitude cloud toward HD 210121 by Gredel et al. (1991).

\section{Evidence for shock chemistry?}

Ever since its identification in 1941, the large abundance of $\mathrm{CH}^{+}$in diffuse clouds has remained a puzzle. The problem stems from the fact that the reaction $\mathrm{C}^{+}+\mathrm{H}_{2} \rightarrow \mathrm{CH}^{+}$ $+\mathrm{H}$ is endoergic by $0.4 \mathrm{eV}$ and therefore does not proceed at low $T$. At the same time, rapid destruction of $\mathrm{CH}^{+}$is ensured in all environments through reactions with $\mathrm{H}_{1} \mathrm{H}_{2}$ or $e$, or by photodissociation. Shocks which heat a narrow layer of the cloud to about $2000 \mathrm{~K}$ therefore seem a promising explanation (Elitzur \& Watson 1978). These shocks should not overproduce molecules such as $\mathrm{CH}$ and $\mathrm{OH}$, however, which are already accounted for in the steady-state models. The most successful shock models have therefore been of the magnetohydrodynamic type, in which part of the energy necessary for overcoming the barrier stems from the differential streaming of the ions and the neutrals (Draine \& Katz 1986; Pineau des Forêts et al. 1986). One interesting feature of the MHD models is that they predict $\mathrm{CH}$ column densities and line widths consistent with those found in the broad components of Lambert et al. (1990). However, there are several recent observational and theoretical developments which cast severe doubts on their validity.

First, the predicted velocity shift between $\mathrm{CH}^{+}$and quiescent $\mathrm{CH}$ or $\mathrm{CN}$ is not observed in recent high-quality data, which indicate $\Delta V<1 \mathrm{~km} \mathrm{~s}^{-1}$ (Crawford 1989; Jenkins et al. 1989; Hawkins \& Craig 1991). Second, the $\mathrm{CH}^{+}$column density continues to increase with $A_{V}$ in translucent clouds (Souza 1979; Gredel et al. 1992). Also, the abundance of the ion tends to be inversely correlated with density, as if its formation is "quenched" at higher densities (Cardelli et al. 1990; Gredel et al. 1992). Third, there are examples of regions 
where the evidence for shocks is very strong but where the $\mathrm{CH}^{+}$column density is very low, such as toward HD 62542 (Cardelli et al. 1990) and in some high-latitude clouds (de Vries \& van Dishoeck 1988). Finally, the latest shock models of Hartquist et al. (1990) suggest that no consistent model can be found that accounts for both $\mathrm{CH}^{+}$, the rotationally-excited $\mathrm{H}_{2}$ and $\mathrm{OH}$. On the other hand, no satisfactory alternative solution to the $\mathrm{CH}^{+}$problem has yet been found. Its broad (but symmetric) line profiles indicate a different formation environment from that of the quiescent $\mathrm{CH}$ and $\mathrm{CN}$, but the absence of velocity shifts and the increase in column density with $A_{V}$ suggest that these two environments are well mixed. A possible solution, originally proposed by Dalgarno (1976) and recently explored by Duley et al. (1992), is that the ion is formed at the turbulent interfaces between the warm atomic and molecular gas (see also Falgarone, this volume). Detailed models for such regions must await a physical description of turbulence.

Large abundances of $\mathrm{OH}$ have also been claimed as evidence for shock chemistry. However, the $\mathrm{OH}$ velocities derived from $18 \mathrm{~cm}$ emission lines also agree within $1 \mathrm{~km} \mathrm{~s}^{-1}$ with those of the quiescent CO (Magnani \& Siskind 1990). Moreover, the abundances found by Magnani \& Siskind do not indicate large enhancements in translucent and high-latitude clouds compared with classical diffuse clouds. The large $\mathrm{OH}$ abundance found in one particular high-latitude cloud (Grossman et al. 1991) most likely results from an underestimate of the $\mathrm{H}_{2}$ column density. $\mathrm{OH}$ column densities up to an order of magnitude larger than found in diffuse clouds can easily be produced in steady-state models in which the radiation field is reduced. In summary, neither the $\mathrm{CH}^{+}$nor the $\mathrm{OH}$ observations appear to support the current shock models.

\section{Evidence for grain-surface chemistry?}

One of the more exciting recent developments in diffuse cloud chemistry is the detection of $\mathrm{NH}$ by Meyer \& Roth (1991) toward $\zeta$ Per. Its abundance is $\mathrm{NH} / \mathrm{H}_{2} \approx 2 \times 10^{-9}$, compared with $\mathrm{CH} / \mathrm{H}_{2} \approx \mathrm{OH} / \mathrm{H}_{2} \approx 5 \times 10^{-8}$. The fact that the $\mathrm{CH}$ and $\mathrm{OH}$ abundances are much larger than that of $\mathrm{NH}$ has been one of the strongest arguments in favor of gas-phase chemistry (Crutcher \& Watson 1976). However, grain-surface chemistry can contribute to the formation of $\mathrm{CH}$ and $\mathrm{OH}$ at the $1 \%$ level, and can dominate the formation of $\mathrm{NH}$. $\mathrm{NH}$ is not easily formed in the gas phase because $\mathrm{N}$ cannot be photoionized, the $\mathrm{N}+$ $\mathrm{H}_{3}^{+}$reaction is slow, and the $\mathrm{N}^{+}+\mathrm{H}_{2}$ reaction is slightly endothermic. The steadystate gas-phase models of van Dishoeck \& Black, using the latest information on the NH photodissociation (Kirby and Goldfield 1991) and the $\mathrm{N}^{+}+\mathrm{H}_{2}$ reaction (Galloway \& Herbst 1989; Le Bourlot 1991), give for the $\zeta$ Per cloud a $\mathrm{NH}$ column density a factor 20 below the observed value. Mann \& Williams (1985) considered the formation of NH resulting from grain surface reactions, either directly or indirectly through formation of $\mathrm{NH}_{3}$ which then photodissociates to NH. The latter model predicts NH column densities similar to those observed. Possible alternative formation schemes of $\mathrm{NH}$ in e.g. shocked or turbulent interface layers still need to be investigated.

Another molecule whose abundance is difficult to reproduce by gas-phase or shock chemistry is $\mathrm{H}_{2} \mathrm{CO}$. Although large column densities for this molecule have been observed in translucent and high-latitude clouds (Magnani et al. 1988; Heithausen et al. 1987; Turner et al. 1989), it must be stressed that its actual abundance is not well determined, since it is not clear observationally how "diffuse" the positions at which $\mathrm{H}_{2} \mathrm{CO}$ is seen really are. Indeed, it is likely that the molecule is not detected by its $2 \mathrm{~cm}$ or $6 \mathrm{~cm}$ absorption lines until $A_{V}>3$ mag. Still, even models with $A_{V} \approx 3-5$ mag fail by several orders of magnitude. The possible formation of $\mathrm{H}_{2} \mathrm{CO}$ on grains in translucent clouds through photoreactions of solid $\mathrm{H}_{2} \mathrm{O}$ and $\mathrm{CO}$ has most recently been investigated by Breukers (1991) and Federman \& Allen (1991). Such models appear capable of reproducing the observed $\mathrm{H}_{2} \mathrm{CO}$ within an order of magnitude, although detailed depth-dependent models have not yet been made. 
Finally, grains affect the chemistry in several indirect ways. The abundances of molecules like $\mathrm{CO}$ and $\mathrm{CN}$ are sensitive to the shape of the ultraviolet extinction curve at the shortest wavelengths (Cardelli 1988; van Dishoeck \& Black 1989). Also, the presence of very small grains or PAHs can affect the ionization balance (Lepp et al. 1988), and the thermal balance (d'Hendecourt \& Léger 1987; Lepp \& Dalgarno 1988; van Dishoeck 1990).

\section{Small-scale structure and evolution}

There is growing evidence that interstellar clouds, including the more diffuse ones, have a very "clumpy" or "fractal" structure (see Falgarone, this volume; Black \& van Dishoeck $1991 b)$. How can this affect the models? Since the ultraviolet radiation can penetrate deeper in a clumpy cloud, it becomes more difficult to build up column densities of molecules such as $\mathrm{CO}$. The effects can be explored in first approximation by using different effective grain scattering parameters (Boissé 1990). Also, large density fluctuations can significantly affect the chemistry of simple molecules such as $\mathrm{C}_{2}, \mathrm{CN}$ and $\mathrm{CO}$, as illustrated by van Dishoeck et al. (1991). The complicated velocity structure, however, may not have much effect, since the damped ultraviolet lines through which $\mathrm{H}_{2}$ and $\mathrm{CO}$ photodissociate are so broad that the various clumps shield each other. This is demonstrated for the case of $\pi$ Sco by models of Jenkins et al. (1989).

A related question is the evolutionary state of diffuse clouds and their relation to dense clouds. It should be realized that at the edges of diffuse and translucent clouds, the chemical time scales are so short, of order $10^{3}-10^{4} \mathrm{yr}$, that chemical equilibrium is readily attained. The same holds for most time scales in the center of the clouds. The major exception is formed by the $\mathrm{H}_{2} / \mathrm{H}$ equilibrium, and possibly by the $\mathrm{CO} / \mathrm{C} / \mathrm{C}^{+}$case in translucent clouds. Time dependent models of the $\mathrm{H}_{2} / \mathrm{H}$ chemistry in diffuse clouds have been presented by Wagenblast \& Hartquist (1989), and indeed, the results depend on whether the hydrogen is initially atomic or molecular. Further study of such evolutionary effects may provide important clues to the origin of diffuse, translucent and high-latitude clouds.

Acknowledgment. The author is grateful to J.H. Black for useful discussions and to the Netherlands Organization for Scientific Research (NWO) for financial support.

Amano, T. 1990, J. Chem. Phys., 92, 6492.

Black, J.H. 1987, in IAU Symposium 120, Astrochemistry, eds. M.S. Vardya and S.P. Tarafdar (Reidel, Dordrecht), p. 217.

Black, J.H. \& Dalgarno, A. 1977, ApJS, 34, 405.

Black, J.H. \& van Dishoeck, E.F. 1991a, ApJ, 369, L9.

Black, J.H. \& van Dishoeck, E.F. 1991b, in Fragmentation of Molecular Clouds and Star Formation, IAU Symposium 147, eds. E. Falgarone et al. (Kluwer, Dordrecht), p. 139.

Blitz, L., Bazell, D., \& Désert, F.X. 1990, ApJ, 352, L13.

Boissé, P., 1990, A\&A, 228, 483.

Breukers, R. 1991, PhD Thesis, University of Leiden.

Cardelli, J.A. 1988, ApJ, 335, 177.

Cardelli, J.A., Suntzeff, N.B., Edgar, R.J. \& Savage, B.D. 1990, ApJ, 362, 551.

Cardelli, J.A. et al. 1991, ApJ, 377, L57.

Crawford, I.A. 1989, MNRAS, 241, 575.

Crutcher, R.M. \& Watson, W.D. 1976, ApJ, 209, 778.

Crutcher, R.M. \& Watson, W.D. 1981, ApJ, 244, 855.

Dalgarno, A. 1976, in Atomic Processes and applications, eds. P.G. Burke and B.L. Moiseiwitsch (North-Holland), p. 110.

Dalgarno, A. 1988, Astro. Lett. and Communications, 26, 153.

de Vries, C.P. \& van Dishoeck, E.F. 1988, A\&A, 203, L23. 
d'Hendecourt, L.B. \& Léger, A. 1987, A\&A, 180, L9.

Draine, B.T. \& Katz, N.S. 1986, ApJ, 306, 655; 310, 392.

Duley, W.W. et al. 1992, MNRAS, in press.

Eidelsberg, M. \& Rostas, F. 1990, A\&A, 235, 472.

Elitzur, M. \& Watson, W.D. 1978, ApJ, 222, L141.

Falgarone, E., Phillips, T.G., \& Walker, C.K. 1991, ApJ, 378, 186.

Federman, S.R. 1982, ApJ, 257, 125.

Federman, S.R. \& Allen, M. 1991, ApJ, 375, 157.

Galloway, E.T. \& Herbst, E. 1989, A\&A, 211, 413.

Gerlich, D. 1989, private communication.

Gredel, R., van Dishoeck, E.F., de Vries, C.P., and Black, J.H. 1991, A\&A, in press.

Gredel, R., van Dishoeck, E.F. \& Black, J.H. 1992, A\&A, submitted.

Grossman, V., Heithausen, A., Meyerdierks, H., \& Mebold, U. 1990, A\&A, 240, 400.

Hartquist, T.W., Flower, D.R., \& Pineau des Forêts, G. 1990, in Molecular Astrophysics -A volume honoring Alexander Dalgarno, ed. T.W. Hartquist (Cambridge University Press).

Hawkins, I. \& Craig, N. 1991, ApJ, 375, 642.

Heithausen, A., Mebold, U. \& de Vries, H.W. 1987, A\&A, 179, 263.

Jenkins, E.B. \& Shaya, E.J. 1979, ApJ, 231, 55.

Jenkins, E.B., Lees, J.F., van Dishoeck, E.F., \& Wilcots, E.M. 1989, ApJ, 343, 785.

Jura, M. 1975, ApJ, 197, 581.

Kirby, K.P. \& Goldfield, E.M. 1991, J. Chem. Phys., 94, 1271.

Lada, E.A. \& Blitz, L. 1988, ApJ, 326, L69.

Lambert, D.L., Sheffer, V., \& Crane, P. 1990, ApJ, 359, L19.

Le Bourlot, J. 1991, A\&A, 242, 235.

Lepp, S. \& Dalgarno, A. 1988, ApJ, 335, 769.

Lepp, S., Dalgarno, A., van Dishoeck, E.F., \& Black, J.H. 1988, ApJ, 329, 418.

Letzelter, C. et al. 1987, Chem. Phys., 114, 273.

Magnani, L., Blitz, L., \& Mundy, L. 1985, ApJ, 295, 402.

Magnani, L., Blitz, L., \& Wouterloot, J.G.A. 1988, ApJ, 326, 909.

Magnani, L. \& Siskind, L. 1990, ApJ, 359, 355.

Mann, A.P.C. \& Williams, D.A. 1984, MNRAS, 209, 33.

Meyer, D.M. \& Roth, K. 1991, ApJ, 376, L49.

Pineau des Forêts, G. et al. 1986, MNRAS, 220, 801.

Pound, M.W., Bania, T.M., \& Wilson, R.W. 1990, ApJ, 351, 165.

Roberge, W.G., Jones, D., Lepp, S., \& Dalgarno, A. 1991, ApJS, 77, 287.

Savage, B.D. et al. 1991, ApJ, 377, L53.

Smith, A.M., Krishna Swamy, K.S. \& Stecher, T.P. 1978, ApJ, 220, 138.

Smith, A.M. et al. 1991, ApJ, 377, L61.

Smith, I.W.M. 1989, ApJ, 347, 282.

Souza, S.P. 1979, PhD thesis, State Univ. of New York at Stony Brook.

Stark, G. et al. 1991, ApJ, 369, 574.

Turner, B.E., Rickard, L.J, \& Xi, L.P. 1989, ApJ, 344, 292.

van Dishoeck, E.F. 1988, in Rate Coefficients in Astrochemistry, eds. T.J. Millar and D.A. Williams (Kluwer, Dordrecht), p. 49.

van Dishoeck, E.F. 1990, in The Evolution of the Interstellar Medium, A.S.P. Conference series nr. 12, ed. L. Blitz (Astronomical Society of the Pacific, San Francisco), p. 207.

van Dishoeck, E.F. \& Black, J.H. 1982, ApJ, 258, 533.

van Dishoeck, E.F. \& Black, J.H. 1986, ApJS, 62, 109.

van Dishoeck, E.F. \& Black, J.H. 1988a, in Rate Coefficients in Astrochemistry, eds. T.J. Millar and D.A. Williams (Kluwer, Dordrecht), p. 209.

van Dishoeck, E.F. \& Black, J.H. 1988b, ApJ, 334, 771.

van Dishoeck, E.F. \& Black, J.H. 1989, ApJ, 340, 273.

van Dishoeck, E.F., Black, J.H., Phillips, T.G., \& Gredel, R. 1991, ApJ, 366, 141.

Viala, Y.P., Roueff, E., \& Abgrall, H. 1988a, A\&A, 190, 215.

Viala, Y.P., Letzelter, C., Eidelsberg, M., \& Rostas, F. 1988b, A\&A, 193, 265.

Wagenblast, R. 1991, preprint.

Wagenblast, R. \& Hartquist, T.W. 1989, MNRAS, 237, 1019. 


\section{QUESTIONS AND ANSWERS}

D.A.Williams: (comments) The failure of diffuse clouds models to account for $\mathrm{CO}$ is particularly disturbing. There is a new work (see Wagenblast's poster) which allows $\boldsymbol{H}_{2}$ (high J) formation on dust, so that the UV field can be relatively low. This work leads to a consistent model of $\zeta O p h$. It suggests that parameter space may not yet be thoroughly explored. A model of $\zeta$ Per with NH formation on dust has been made by Wagenblast \& Williams. It gives results entirely consistent with all atomic and molecular observations on this line of sight. The failure of shocks to account satisfactorily for $\mathrm{CH}^{+}$seems to be established. An alternative (suggested by A.Dalgarno) is that $\mathrm{CH}^{+}$may be formed in warm interface region. An exploratory calculation (see Poster: Duley et al.) indicates that such a solution is possible without contravening the constraints of $\mathrm{H}_{2}(\mathrm{~J}), \mathrm{OH}$ and $\mathrm{CH}$.

E.F.van Dishoeck: I agree that the $\mathrm{H}_{2}$ formation model may be important for the population of the higher $\mathrm{J}$ levels, an effect which was already explored to some extent by Black and Dalgarno (1976) and van Dishoeck and Black (1986). See also van Dishoeck 1990 (in "The evolution of the ISM", ed. L.Blitz) for a model for the $\zeta O p h$ cloud with $I_{u v}=1$. The problem is how to distinguish between the models; the UV pumping models make specific predictions about the vibrationally excited $\mathrm{H}_{2}$. Note also that the high UV field models only fail for $\zeta O p h$, but not for $\zeta P e r$ etc if a steep rising UV extinction curve is taken into account. Regarding $\mathrm{CH}^{+}$, I fully agree that warm interfaces are a likely site for the formation of the ion. However, until there is a physical model for turbulence, we cannot prove anything quantitatively. 